\title{
Compromiso organizacional y su influencia en la satisfacción laboral de los colaboradores de la empresa de transportes Móvil Tours S.A.C. - Agencia Chachapoyas, 2016
}

\section{Organizational commitment and its influence on the job satisfaction of the employees of the transport company Móvil Tours S.A.C. - Chachapoyas Agency, 2016}

\author{
Angélica Rosario Terrones Villegas ${ }^{1}$
}

\begin{abstract}
RESUMEN
La presente investigación tuvo por objetivo Determinar la influencia del compromiso organizacional en la satisfacción laboral de los colaboradores de la empresa de transportes Móvil Tours S.A.C. - agencia Chachapoyas. Para el desarrollo de la investigación se hizo uso de los métodos deductivo e inductivo que permitió el acopio de información secundaria, de fuentes bibliográficas que permitieron elaborar el marco teórico de la investigación. La muestra de estudio estuvo conformada por 18 colaboradores de la empresa Móvil tours S.A.C. - agencia Chachapoyas. El muestreo aplicado fue el no probabilístico accidental. Para la recolección de datos se utilizó la técnica del análisis documenta, análisis descriptivo y análisis cuantitativo. En los resultados se muestra que la satisfacción laboral de los colaboradores de la Empresa de Transportes Móvil Tours S.A.C. Agencia Chachapoyas es de poco satisfecho a regularmente satisfecho; existiendo un déficit que repercute en el cumplimiento de las funciones del personal y que se refleja en el servicio que se ofrece. Concluyendo que la prueba estadística aplicada al estudio arroja como resultado la relación entre las variables compromiso organizacional y satisfacción laboral entendiéndose que un mal compromiso organizacional estaría influyendo en poca satisfacción laboral de los colaboradores de la Empresa de Transportes Móvil Tours S.A.C. Agencia Chachapoyas, llegándose a contrastar favorablemente la hipótesis.
\end{abstract}

Palabras claves: compromiso organizacional, influencia, satisfacción laboral, colaboradores.

\begin{abstract}
The objective of the present investigation was to determine the influence of organizational commitment on the job satisfaction of the employees of the transport company Móvil Tours S.A.C. - Chachapoyas agency. For the development of the research, deductive and inductive methods were used, which allowed the collection of secondary information, from bibliographic sources that allowed to elaborate the theoretical framework of the research. The study sample consisted of 18 employees of the company Mobile Tours S.A.C. - Chachapoyas agency. The applied sampling was the accidental non-probabilistic one. For data collection, the technique of document analysis, descriptive analysis and quantitative analysis were used. The results show that the job satisfaction of the employees of the Transport Company Mobile Tours S.A.C. Chachapoyas Agency is from little satisfied to regularly satisfied; there is a deficit that affects the fulfillment of the functions of the staff and that is reflected in the service offered. Concluding that the statistical test applied to the study results in the relationship between the variables organizational commitment and job satisfaction understanding that a bad organizational commitment would be influencing low job satisfaction of the employees of the Transport Company Mobile Tours S.A.C. Chachapoyas Agency, coming to contrast the hypothesis favourably.
\end{abstract}

Keywords: organizational commitment, influence, job satisfaction, employees.

${ }^{1}$ Licenciada en Turismo y Administración; Bachiller en Turismo y Administración. angelicartv@gmail.com 


\section{INTRODUCCIÓN}

El comportamiento organizacional es un campo del conocimiento humano extremadamente sensible a ciertas características de las organizaciones y de su entorno. Por tanto, es una disciplina que depende de las contingencias y las situaciones, así como de la mentalidad que existe en cada organización y de la estructura organizacional que se adopte como plataforma para las decisiones y las operaciones. Además, también está influida por el contexto ambiental, el negocio de la organización, sus procesos internos, el capital intelectual involucrado e innumerables variables importantes más. Y depende en gran medida de las personas que participan en cada organización. (Mota, 2014)

El compromiso organizacional se ha convertido en una de las variables más estudiadas en el mundo laboral. Una de las razones fundamentales de que esto haya sucedido, es que varias investigaciones han podido demostrar que el compromiso con la organización suele ser un mejor predictor de la eficiencia y de la puntualidad, que cualquier otra variable. Quizás más importantes aún, han sido las evidencias de que las organizaciones cuyos integrantes poseen niveles altos de compromiso son aquéllas que registran altos niveles de desempeño y productividad, y bajos índices de ausentismo. (Arceniega, 2002)

La satisfacción laboral, según manifiestan la mayor parte de los investigadores del comportamiento organizacional, es una actitud y ésta refleja el sentimiento de las personas respecto a algo. Por tal razón se acepta que la satisfacción laboral es la actitud que asume la persona respecto a su trabajo. Si la persona está muy satisfecha, en términos laborales adopta actitudes positivas ante el trabajo y viceversa. (Atalaya, 1999)

La satisfacción laboral de los trabajadores, puede considerarse como un fin en sí misma, que compete tanto al trabajador como a la empresa; que además de producir beneficios a los empleados al ayudarles a mantener una buena salud mental, puede contribuir a mejorar la productividad de una empresa y con ello su rentabilidad; ya que un trabajador motivado y satisfecho está en mejores condiciones de desempeñar un trabajo adecuado, que otro que no lo esté.

Recientemente en la empresa de transportes Móvil Tours - Agencia Chachapoyas, se viene apreciando un clima laboral inadecuado, donde los trabajadores actúan sin motivación alguna y los problemas entre ellos se reflejan en el desarrollo ineficaz de su trabajo. Esto se debe en gran parte a la insatisfacción que presentan las personas con respecto a su trabajo, no se sienten a gusto con la forma en que deben desempeñarse, ven su labor como algo rutinario, no se sienten motivados lo suficiente como para laborar mejor, entre otros aspectos; todo ello trae como consecuencia un mal trato al público y el decrecimiento de la institución. (Gonzales, 2015)

Teniendo en cuenta que la misión de la empresa móvil tours es brindar soluciones integrales y eficientes en transporte, orientadas a la satisfacción del cliente, surge la necesidad de considerar la importancia del compromiso organizacional y la satisfacción laboral como factores determinantes en la eficacia de los colaboradores de la empresa de transportes Móvil Tours S.A.C; a fin de elevar los niveles de productividad y excelencia, propiciando además una verdadera identificación institucional que sea motivo de satisfacción en todos sus aspectos.

Por tanto este estudio se encaminó a dilucidar el siguiente problema: ¿Qué influencia tiene el compromiso organizacional en la satisfacción laboral de los colaboradores de la empresa de transportes Móvil Tours S.A.C. - Agencia Chachapoyas, 2016?, cuya hipótesis de estudio fue: El Clima Organizacional tiene influencia negativa en la Satisfacción Laboral de los colaboradores de la empresa de transportes Móvil Tours S.A.C. - agencia Chachapoyas, 2016.

\section{MATERIALES Y MÉTODOS}

El diseño de investigación utilizado fue No Experimental-Transeccional.

\section{Muestra}

M1: Se aplicó el muestreo no probabilístico "accidental", y la muestra queda definida de la siguiente manera:

\begin{tabular}{|l|c|}
\hline \multicolumn{1}{|c|}{ DETALLE } & CANT. \\
\hline \begin{tabular}{l|c|} 
Nivel Administrativo de la empresa Móvil \\
Tours - Agencia Chachapoyas (1 \\
Administrador y 1 Asistente
\end{tabular} & 2 \\
Administrativo). & \\
\hline Área de Counter (3 Counter). & 3 \\
\hline Área de Encomiendas (3 Counter, 4 \\
Auxiliares y 2 de Reparto) & 9 \\
\hline Área de Limpieza & 2 \\
\hline Área de Vigilancia & 2 \\
\hline \multicolumn{1}{|c|}{ TOTAL } & $\mathbf{1 8}$ \\
\hline
\end{tabular}

\section{Métodos y técnicas}

El método que se utilizó fue deductivo - inductivo, 
porque se partió de la generalización de la base teórica sobre comportamiento organizacional, para contrastar con la realidad y llegar a probar la hipótesis.

Las técnicas utilizadas fueron:

- Análisis Documental: Teniendo como fuentes a libros, guías, manuales, artículos, revistas, tesis. Análisis Descriptivo: De los datos utilizando estadística descriptiva (cuadros, gráficos de frecuencias y porcentajes).

- Análisis Cuantitativo: Se utilizará la recolección, análisis e interpretación de datos para contestar la pregunta de investigación y probar la hipótesis.

\section{RESULTADOS}

3.1. Resultados del cuestionario aplicado a los colaboradores de la empresa Móvil tours S.A.C. agencia Chachapoyas

Tabla $\mathrm{N}^{\mathrm{o}}$ 01: Edad, sexo, estado civil y procedencia tiempo de los trabajadores de la Empresa de Transportes Móvil Tours S.A.C. Agencia Chachapoyas, 2016.

\begin{tabular}{|c|c|c|}
\hline Edad & fi & Porcentaje (\%) \\
\hline $\begin{array}{l}\text { Menor de } 20 \\
\text { años }\end{array}$ & 1 & 5.6 \\
\hline De 21 a 25 años & 6 & 33.3 \\
\hline De 26 a 30 años & 4 & 22.2 \\
\hline De 31 a 40 años & 5 & 27.8 \\
\hline De 41 a más & 2 & 11.1 \\
\hline Total & 18 & 100 \\
\hline Sexo & fi & Porcentaje (\%) \\
\hline Masculino & 9 & 50 \\
\hline Femenino & 9 & 50 \\
\hline Total & 18 & 100 \\
\hline Estado civil & fi & Porcentaje (\%) \\
\hline Soltero & 9 & 50 \\
\hline Conviviente & 4 & 22.2 \\
\hline Casado & 5 & 27.8 \\
\hline Total & 18 & 100 \\
\hline Procedencia & fi & Porcentaje (\%) \\
\hline $\begin{array}{l}\text { De } \\
\text { Chachapoyas }\end{array}$ & 7 & 38.8 \\
\hline $\begin{array}{l}\text { De otras } \\
\text { provincias de } \\
\text { Amazonas }\end{array}$ & 7 & 38.8 \\
\hline $\begin{array}{l}\text { De otras } \\
\text { regiones }\end{array}$ & 4 & 22.2 \\
\hline Total & 18 & 100 \\
\hline
\end{tabular}

En el cuadro $\mathrm{N}^{\circ} 01$, se muestra que el $33.3 \%$ están en las edades de 21 a 25 y el $27 \%$ entre 31 a 40 . Con respecto al sexo el 50\% son del sexo masculino y el $50 \%$ del sexo femenino. En el estado civil se muestra que el $50 \%$ son solteros y el $27.8 \%$ son casados. En cuanto al lugar de procedencia el 38.8 son de Chachapoyas y el $38.8 \%$ son de otras provincias.

Figura $\mathrm{N}^{\mathrm{o}}$ 01: Compromiso organizacional de los trabajadores de la Empresa de Transportes Móvil Tours S.A.C.Agencia Chachapoyas, 2016

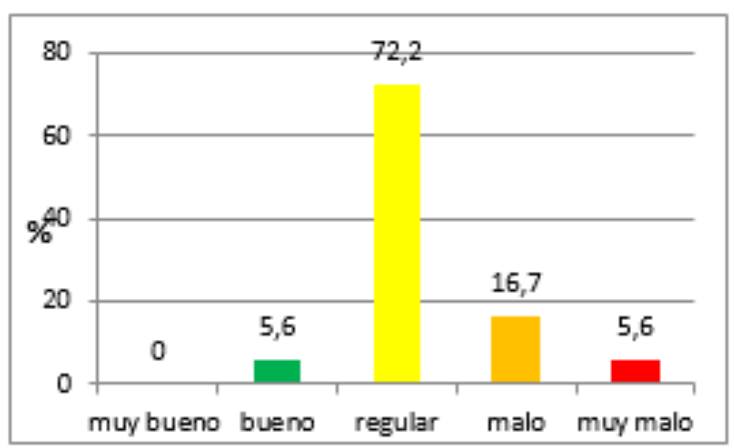

En la figura $\mathrm{N}^{\circ} 01$, se muestra que el compromiso organizacional de los trabajadores de la empresa es regular con un $72.2 \%$ y el $16.7 \%$ es malo.

Figura $\mathrm{N}^{\circ}$ 02: Compromiso organizacional según dimensiones en los trabajadores de la Empresa de Transportes Móvil Tours S.A.C. Agencia Chachapoyas, 2016.

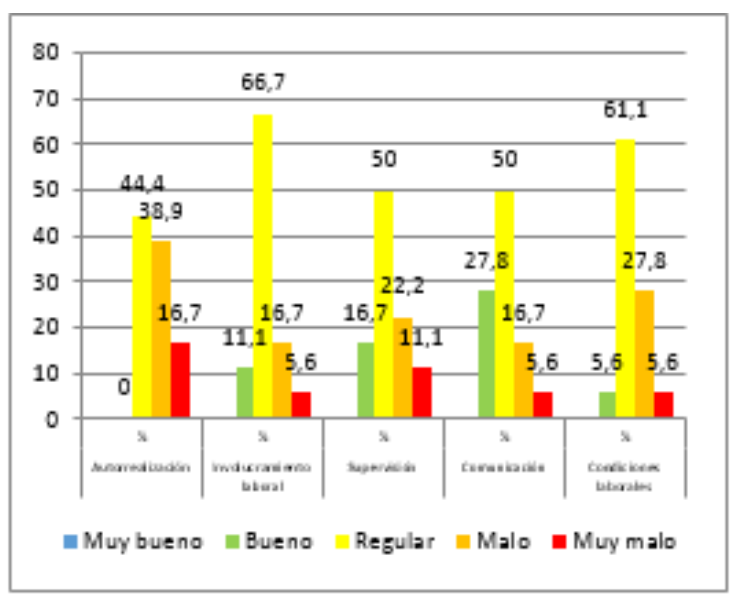

En la figura $\mathrm{N}^{\circ} 02$, se muestra que el $44.4 \%$ considera que la posibilidad de autorrealización es regular frente a la dimensión autorrealización, el $66.7 \%$ percibe un involucramiento laboral regular frente a la dimensión involucramiento laboral, el $50 \%$ percibe que la supervisión es regular en la dimensión Supervisión, 50\% percibe una comunicación regular en la empresa en la dimensión Comunicación y $61.1 \%$ percibe regulares condiciones laborales en la dimensión Comunicación.

Figura $\mathrm{N}^{\mathrm{o}}$ 03: Motivación en el trabajo en los 
trabajadores de la Empresa de Transportes Móvil Tours S.A.C.Agencia Chachapoyas, 2016.

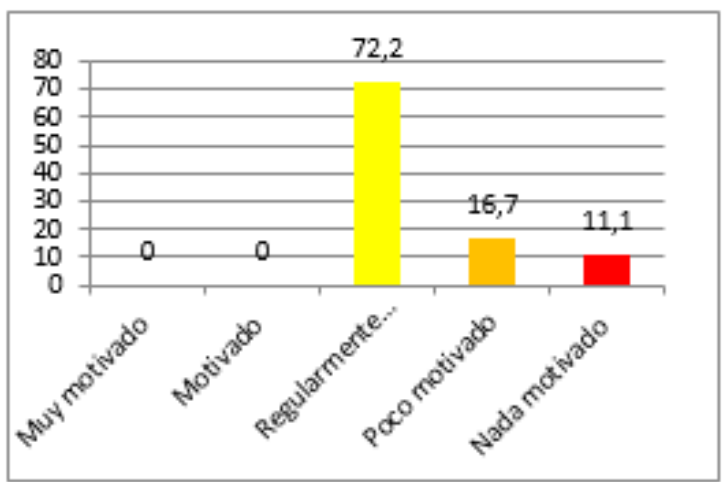

En la figura $\mathrm{N}^{\circ} 03$, se muestra que el $72.2 \%$ de los trabajadores se encuentra regularmente motivado.

Figura $N^{\circ}$ 04: Satisfacción laboral en los trabajadores de la Empresa de Transportes Móvil Tours S.A.C. Agencia Chachapoyas, 2016.

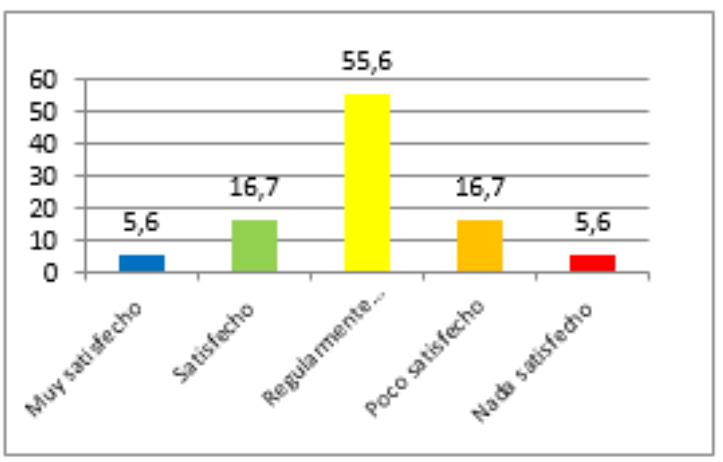

En la figura $\mathrm{N}^{\mathrm{o}} 04$, se muestra que el $55.6 \%$ de los trabajadores están regularmente satisfechos.

Figura $\mathrm{N}^{\mathrm{o}}$ 05. Relación entre el Compromiso Organizacional y la Satisfacción Laboral en los trabajadores de la Empresa de Transportes Móvil Tours S.A.C. Agencia Chachapoyas, 2016

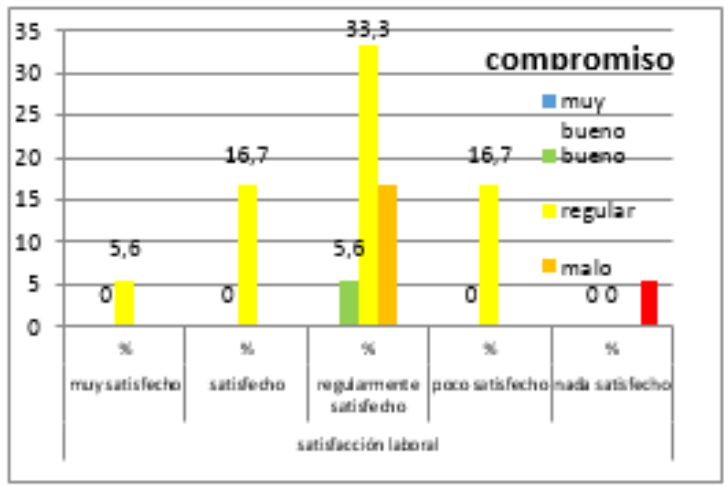

En la figura $\mathrm{N}^{\mathrm{o}} 05$, se muestra que el $33.3 \%$ de los encuestados considera que el compromiso organizacional es regular y al mismo tiempo tienen un satisfacción laboral regular.
Figura $\mathrm{N}^{\circ}$ 06: Relación entre Motivación en el Trabajo y Satisfacción Laboral en los trabajadores de la Empresa de Transportes Móvil Tours S.A.C. Agencia Chachapoyas, 2016.

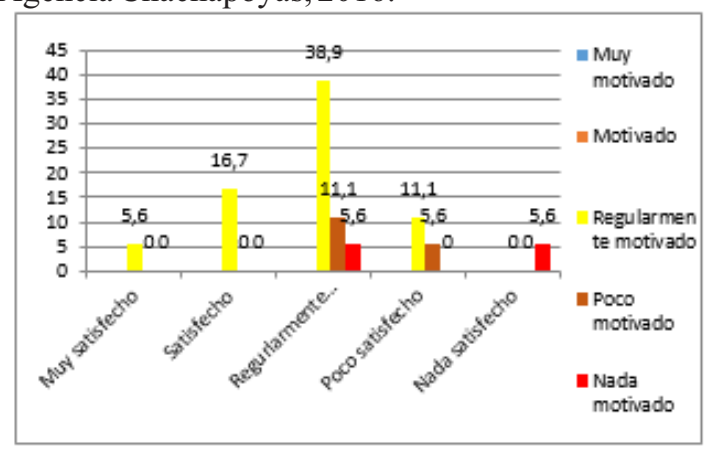

En la figura $\mathrm{N}^{\circ} 06$, se muestra que el $38.9 \%$ de los trabajadores están regularmente motivados y al mismo tiempo regularmente satisfechos.

Figura $\mathrm{N}^{\circ}$ 07. Relación entre Compromiso Organizacional y Motivación en el Trabajo en los trabajadores de la Empresa de Transportes Móvil Tours S.A.C. Agencia Chachapoyas, 2016.

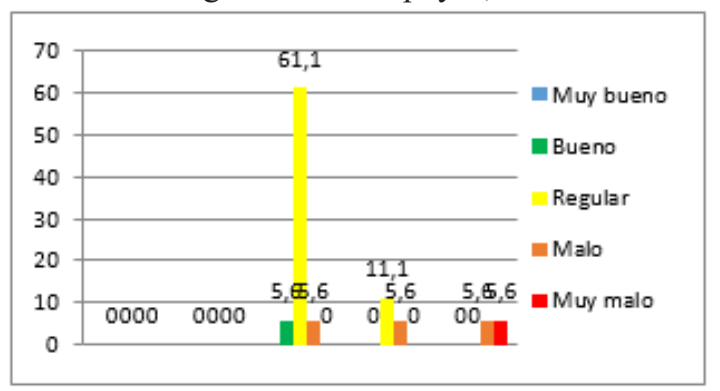

En la figura $\mathrm{N}^{\circ} 07$, se muestra que el $61.1 \%$ de los encuestados considera que el compromiso organizacional es regular y al mismo tiempo se encuentran regularmente motivados.

\section{DISCUSIÓN}

La investigación se orienta en dos variables de investigación que son: el compromiso organizacional y la satisfacción laboral. Se debe entender que la primera variable forma parte de la teoría del comportamiento organizacional a la que Chiavenato define como el estudio de las personas y a los grupos que actúan en las organizaciones. Bajo esta premisa la investigación estudió el compromiso organizacional del recurso humano que labora en la empresa de transportes Móvil Tours S.A.C. - Agencia Chachapoyas; como parte de la investigación se determinó la influencia que los trabajadores ejercen en esta organización guardando congruencia y similitud con la teoría de Chiavenato.

Con respecto a la variable satisfacción laboral, Ardoin lo teoriza como la actitud del trabajador frente 
a su propio trabajo. Las actitudes son determinadas conjuntamente por las características actuales del puesto como por las percepciones que tiene el trabajador de lo que deberían ser. En la investigación se han aplicado técnicas como la encuesta que han permitido obtener información sobre las actitudes del trabajador con relación a su trabajo y sus percepciones del cómo debería ser; cumpliéndose con la teoría propuesta por Ardoin.

Marroquín y Pérez, en la investigación denominado el clima organizacional y su relación con el desempeño laboral en los trabajadores de Burger King, se plantean como objetivo describir la relación entre clima organización y el desempeño laboral de los trabajadores de Burger King y así mismo identificar el nivel del clima organización en los trabajadores de la empresa. A estos objetivos de la investigación con la presente tesis se puede discutir que existe relación considerando que el objetivo principal es determinar la influencia del compromiso organización en la satisfacción laboral y uno de los objetivos específicos está relacionado con la determinación del nivel del clima organizacional. También se puede discutir los métodos utilizados para la investigación, utilizan el método cuantitativo para evaluar los procesos y actividades en su área de estudio, la presente investigación utilizó los métodos deductivo inductivo por que se partió de la elaboración de la base teorica para constrastar el comportamiento organizacional con al realidad; también fue utilizado el método cuantitativo en el procesamiento, presentación y análisis de datos que han permitido identificar la relación entre las dos variables compromiso organizacional y satisfacción laboral y cada uno de los niveles propuestos en los objetivos específicos.

Hinojosa, 2010; en su tesis clima organizacional y satisfacción laboral de profesores del Colegio Sagrados Corazones Padres Franceses, evalúa y describe el clima organizacional. Esta investigación guarda relación con la presente teniendo como incongruencia solamente el ámbito donde se aplica, ya que el primero se da en una Institución Educativa y la segunda en una empresa de transportes Interprovincial, ambas ofertan servicios. Hinojosa establece como objetivo evaluar y describir el compromiso organizacional que existe en el colegio Sagrado Corazón para proveer información que permita develar el nivel de satisfacción laboral de los profesores que trabajan en el colegio; tanto que el objetivo de la presente tesis es determinar la influencia del compromiso organizacional en la satisfacción laboral de los colaboradores de la empresa de transportes Móvil Tours Chachapoyas, ambas investigaciones parten de un diagnóstico que les a permitido describir sus variables dentro de un contexto problemático y esto coincide con los objetivos de las investigaciones; considerando que la tesis de la empresa Móvil Tours plantea como uno de sus objetivos específicos determinar el nivel de compromiso organizacional, se debe tener en cuenta que estas forman parte de una relación vinculada al comportamiento de los trabajadores en un contexto con características que promueven estas actitudes. Dentro de los objetivos específicos ambas investigaciones se proponen determinar el nivel de satisfacción laboral de sus colaboradores, teniendo en cuenta que esta repercute en la calidad de servicio que brindan estas empresas en el sector que pertenecen.

A nivel nacional Pelaes, O. (2010). en su tesis denominado relación entre el clima organizacional y la satisfacción del cliente en una empresa de servicios telefónicos, concluye que existe una influencia directa entre clima laboral y la satisfacción del cliente y viceversa. En la presente tesis la prueba estadística aplicada al estudio arroja como resultado la relación entre las variables compromiso organizacional y satisfacción laboral entendiéndose que un mal compromiso organizacional estaría influyendo en poca satisfacción laboral de la empresa móvil tours. Ambas hipótesis han sido contrastadas favorablemente comprobándose que las relaciones interpersonales, estilo de dirección el sentido de pertenencia, de retribución, la estabilidad, la claridad y coherencia en la dirección se relacionan significativamente, tanto con el cliente como es el caso de Peleas León, como con los trabajadores para la presente tesis.

Las investigaciones de Marroquín Pérez, Hinojoza y Peláez, discutidas con la presente tesis, se vinculan a esta investigación en el enfoque teórico de clima organizacional que son el conjunto de características que condicionan a una organización y que influyen en su producción sea de bienes o servicios. Las investigaciones también se vinculan bajo el enfoque de satisfacción laboral que es un estado producto del compromiso organizacional y que va repercutir en la satisfacción de los clientes de las empresas objeto de estudio.

En la investigación se propuso como objetivo específico determinar el nivel de compromiso organizacional de los colaboradores en la empresa Móvil Tours, esto queda determinado en la tabla 1 de resultados, donde el $88 \%$ de los encuestados se encuentra en los niveles regular a malo evidenciando que existe un déficit en esta variable. En relación al objetivo específico que determina el nivel de satisfacción laboral en la tabla 4 se determinó que los 
trabajadores están regularmente satisfechos en un $55 \%$ y poco satisfecho en $3 \%$ concluyéndose que el nivel de satisfacción es regular. Bajo estos resultados del análisis de la influencia del compromiso organizacional en la satisfacción laboral se puede concluir que existe una relación directa y que repercute guardando relación con la hipótesis que sostiene de que la influencia es negativa en la satisfacción laboral.

La investigación se enmarca dentro de las líneas de la investigación denominada promoción y desarrollo de empresas competitivas, por las características propias el ámbito de estudio y las variables a investigar que son el compromiso organizacional y la influencia en la satisfacción laboral, que repercuten directamente en la competitividad y éxito de la empresa; en tal sentido se considera a esta línea de investigación acorde con el trabajo realizado.

En el área de estudio se han podido identificar otra serie de problemas que pueden ser materia de investigaciones futuras como la atención al cliente, fortalecimiento de capacidades, mejora de la rentabilidad de la empresa y estrategias de marketing a las cuales se les podría aplicar como hipótesis posibles de investigación que para mejorar lo identificado es necesario establecer lineamientos planificados orientados a la mitigación de los problemas enunciados.

Para la presente tesis el problema principal radicó en la identificación del instrumento estadístico adecuado que permita medir la relación entre las variables compromiso organizacional y satisfacción laboral, lográndose solucionar esto a través del programa software SPSS versión 23 Microsoft Word y la hoja de cálculo Excel; para contrastar la hipótesis se utilizó la prueba estadística no paramétrica de la Ji - cuadrado con nivel de satisfacción de $\&=0.05$ ( $95 \%$ de confiabilidad y $5 \%$ de margen de error). La presentación de los resultados se realizó en tablas simples, tablas de contingencia y figura de barras.

\section{CONCLUSIONES}

Se ha determinado que el nivel de compromiso organizacional de los colaboradores de la empresa de transportes Móvil Tours S.A.C. - agencia Chachapoyas es de regular a malo; los colaboradores perciben un ambiente laboral deficiente, reflejado en factores como: rendimiento, productividad y satisfacción.

Se determinó que el nivel de satisfacción laboral que de los colaboradores de la Empresa de Transportes Móvil Tours S.A.C. Agencia Chachapoyas es de poco satisfecho a regularmente satisfecho; existiendo un déficit que repercute en el cumplimiento de las funciones del personal y que se refleja en el servicio que se ofrece.

Existe una relación directa entre el compromiso organizacional y la satisfacción laboral; identificándose un déficit que trae como consecuencias un regular y deficiente compromiso organizacional que repercute en la prestación del servicio de los colaboradores de la Empresa de Transportes Móvil Tours S.A.C. Agencia Chachapoyas.

La prueba estadística aplicada al estudio arroja como resultado la relación entre las variables compromiso organizacional y satisfacción laboral entendiéndose que un mal compromiso organizacional estaría influyendo en poca satisfacción laboral de los colaboradores de la Empresa de Transportes Móvil Tours S.A.C. Agencia Chachapoyas, llegándose a contrastar favorablemente la hipótesis.

\section{REFERENCIAS BIBLIOGRÁFICAS}

Arceniega, L. (2002). Compromiso organizacional en Mexico.

Atalaya, M. (1999). Satisfacción laboral y productividad. Obtenido de http://sisbib.unmsm.edu.pe/bvrevistas/p sicologia/1999_n5/satisfaccion.htm

Gonzales, J. y. (2015). Aplicación de un programa de motivación para incrementar el nivel de satisfacción laboral de los trabajadores de la empresa agrotransportes gonzales s.r.l de la ciudad de trujillo, periodo abril-junio del año 2015. Obtenido de http://repositorio.upao.edu.pe/bitstream/ upaorep/1427/1/Gonzales_Segundo_Mo tivacion_Incrementar-Laboral.pdf

Hinojosa, C. (2010). Clima organizacional y satisfacción laboral de profesores del colegio sagrados corazones padres franceses. Chile.

Marroquín, S. y Pérez, L. (2011). El clima organizacional y su relación con el desempeño laboral. Guatemala.

Mota, J. (2014). Comportamiento organizacional Chiavenato. Obten ido d e http://www.academia.edu/9000927/Com portamiento_Organizacional_Chiavenat o

Pelaes, O. (2010). Relación entre el clima organizacional y la satisfacción del cliente en una empresa de servicios telefónicos. lima. 